\title{
Evaluation of Nine Low-cost-sensor-based Particulate Matter Monitors
}

\author{
Jiayu Li ${ }^{1 \#}$, Simar K. Mattewal ${ }^{1,2 \#}$, Sameer Patel ${ }^{1,3}$, Pratim Biswas ${ }^{1^{*}}$ \\ ${ }^{1}$ Aerosol and Air Quality Research Laboratory, Department of Energy, Environmental and Chemical Engineering, \\ Washington University in St. Louis, St. Louis, MO 63130, USA \\ ${ }^{2}$ Indian Institute of Technology Delhi, New Delhi 110016, India \\ ${ }^{3}$ University of Colorado, Boulder, CO 80309, USA
}

\begin{abstract}
Due to their affordability, compact size, and moderate accuracy, low-cost sensors have been studied extensively in recent years. Different manufacturers employ different calibration methodologies and provide users with calibration factors for their models. This study assessed the performance of nine low-cost PM monitors (AirVisual, Alphasense, APT, Awair, Dylos, Foobot, PurpleAir, Wynd, and Xiaomi) in a chamber containing a well-defined aerosol. A GRIMM and a SidePak were used as the reference instruments. The monitors were divided into two groups according to their working principle and data reporting format, and a linear correlation factor for the $\mathrm{PM}_{2.5}$ mass concentration measurement was calculated for each monitor. Additionally, the differences between the mass concentrations reported by the various monitors and those measured by the reference instruments were plotted against their average before and after user calibration to demonstrate the degree of improvement possible with calibration. Bin-specific calibration was also performed for monitors reporting size distributions to demonstrate coincidence errors that could bias the results. Since monitors designed for residential use often display the air quality index, typically illustrating it with a simplified, colorcoded index, the color schemes of various monitors were evaluated against the U.S. EPA regulation to determine whether they could convey the overall air quality accurately and promptly. Although these residential monitors indicated the air quality moderately well, their differing color schemes made the evaluation difficult and potentially inaccurate. Altogether, the tested monitors offer low-cost sensors in packages that are convenient for use and ready for deployment without additional assembly. However, to improve the accuracy of the measurements, user-defined calibration for the target PM source is still recommended.
\end{abstract}

Keywords: Low-cost PM monitors; Residential application; Calibration and characterization; Air quality index.

\section{INTRODUCTION}

As a major atmospheric pollutant, particulate matter (PM) has adverse health effects; therefore, it is routinely monitored to reduce the harm it causes. In 2013, PM was classified as a Group 1 carcinogen by the International Agency for Research on Cancer (IARC), and has been associated with lung cancer, premature mortality, cardiopulmonary diseases, and cardiovascular diseases (Valavanidis et al., 2008; Brook et al., 2010; Stewart and Wild, 2017). PM pollution accounts for nearly 1.2 million premature deaths and approximately 6.4 million annual deaths globally (Smith and Mehta, 2003;

\footnotetext{
\# These authors contributed equally to this work.

* Corresponding author.

Tel.: +1-314-935-5548; Fax: +1-314-935-5464

E-mail address: pbiswas@wustl.edu
}

Cohen et al., 2005; Burnett et al., 2014). It has been reported that when the mass concentration of fine particulate matter $\left(\mathrm{PM}_{2.5}\right.$, particulate matter with a diameter $\left.<2.5 \mu \mathrm{m}\right)$ increases by about $10 \mu \mathrm{g} \mathrm{m}^{-3}$, the risk of lung cancer mortality increases by approximately $8 \%$ (Pope III et al., 2002). Due to these adverse health effects, stringent regulations on PM mass concentration have been enacted and enforced by governments and local agencies. The standards for the annual $\mathrm{PM}_{2.5}$ concentration were set as $12 \mu \mathrm{g} \mathrm{m}^{-3}$ and $10 \mu \mathrm{g} \mathrm{m}^{-3}$ by the United States Environmental Protection Agency (U.S. EPA) and the World Health Organization (WHO) respectively (WHO, 2005). Apart from the PM concentration standard, the U.S. EPA also specified the gravimetric method as the Federal Reference Method (FRM) for PM mass concentration measurement. The gravimetric method calculates the PM mass concentration by weighing the particles accumulated on a filter over a period of time. This conventional method has been considered to be one of the most reliable methods for PM mass concentration measurement; nevertheless, maintaining the system and 
weighing filters are laborious. Furthermore, the gravimetric method is not real-time measurement and can report only the accumulative PM mass concentration. The cost and labor required by the gravimetric method have hindered the application of this method for personal use. Alternative measurement methods, for example, TEOM, GRIMM, FIMS, and SMPS, can measure real-time PM concentration accurately, but still face the challenges of high cost and long-term stability (Allen et al., 1997; Sioutas, 1999; Klepeis et al., 2007; Wang et al., 2018).

As a potential alternative method for PM concentration measurement, low-cost PM sensors have been studied extensively in recent years (White et al., 2012; Kumar et al., 2015; Rai et al., 2017; Morawska et al., 2018). Compared to bulky laboratory instruments costing up to thousands of dollars, palm-sized low-cost sensors usually cost less than fifty dollars. It should be noted that the term "low-cost PM sensor" refers only to the opto-electrical sensing module, such as popular models made by Sharp, Shinyei, Samyoung, Oneair, and Plantower (Wang et al., 2015; Sousan et al., 2016b; Kelly et al., 2017; Liu et al., 2017). To make low-cost PM sensors functional, circuit board design, programming, and calibration are necessary to establish the relationship between electrical signals (current, voltage, or pulse width) and the PM concentrations. After assembly and laboratory calibration, low-cost PM sensors exhibit good linearity against reference instruments, showing high potential for personal PM monitors and sensor networks (Wang et al., 2015).

Currently, research on low-cost PM sensors mainly focuses on two aspects: interpreting their signal comprehensively through calibration or characterization and modifying them for personal or sensor network use. Several groups have tried to explain the deficiencies and limitations of low-cost sensors due to their working principles ( $\mathrm{Li}$ and Biswas, 2017; Zhang et al., 2018). Some studies also calibrated a variety of low-cost PM sensors for different aerosol sources (e.g., NaCl, Arizona Road Dust [ARD], sucrose, silica, welding fumes, and diesel fumes) in different test environments (laboratory, residential, and ambient) to identify the optimal performance and favorable working conditions of each type of sensor (Wang et al., 2015; Manikonda et al., 2016; Sousan et al., 2016a, b; Kelly et al., 2017; Liu et al., 2017; Rai et al., 2017; Zikova et al., 2017; Aliyu and Botai, 2018; Johnson et al., 2018; Hapidin et al., 2019). These studies demonstrated the advantages and limitations of these sensors and laid a solid foundation for further deployment and application. Wireless sensors or sensor networks, as one major application, have been deployed in households, meeting rooms, factories, cities, etc. to monitor the dynamic process of pollution events with high spatiotemporal resolution (Kim et al., 2010; Kim et al., 2014; Rajasegarar et al., 2014; Leavey et al., 2015; Patel et al., 2017; Jeon et al., 2018; Li et al., 2018). Some of these studies have explored in depth the algorithms for organizing sensor data and extracting the maximum effective information (He et al., 2018; Li et al., 2018). Apart from use in sensor networks, low-cost PM sensors find another important application as low-cost PM monitors, as we elaborate in the following section.

Low-cost PM monitors, whose major components are lowcost PM sensors, are usually assembled and pre-calibrated before distribution to users. Compared to conventional PM measurement instruments, they still have an appealing price advantage; however, accuracy is still a major concern. Compared to just the PM sensors, the assembled monitors' price is higher, but these monitors are advertised with enhanced data quality and stability due to improved algorithms and advanced factory calibration. Occasionally, these monitors have even been chosen as reference instruments to calibrate low-cost sensors. The Air Quality Sensor Performance Evaluation Center (AQ-SPEC), a unit of the South Coast Air Quality Management District (SCAQMD), has evaluated the majority of commercial monitors for multiple sources (Polidori et al., 2017). They have also built a calibration chamber that can maintain a stable and reproducible test environment (Papapostolou et al., 2017). Several popular monitors have been highlighted recently. The Dylos DC1700 Air Quality Monitor (Dylos Corp., Riverside, CA, USA) has been evaluated for different scenarios and has been deployed in indoor and outdoor environments (Semple et al., 2013; Holstius et al., 2014; Dacunto et al., 2015; Manikonda et al., 2016; Rai et al., 2017). The Alphasense OPC-N3 particulate monitor (Alphasense Ltd., Great Notley, UK) has been evaluated focusing on its ability to accurately report the mass concentration of $\mathrm{PM}_{1}, \mathrm{PM}_{2.5}$, and $\mathrm{PM}_{10}$ (Sousan et al., 2016a; Crilley et al., 2018). The PurpleAir PA-II-SD Air Quality Sensor (PurpleAir, UT, USA) has demonstrated good linearity against reference instruments for both laboratory calibration and ambient field measurement (Kelly et al., 2017). Several other low-cost monitors have also been evaluated and compared in different studies, including the AirVisual Node (AirVisual, Inc., USA), APT low-cost monitor (Applied Particle Technology, MO, USA), Awair air quality monitor (Bitfinder, Inc., CA, USA), Foobot (Airboxlab, San Francisco, CA, USA), wearable Wynd Air Quality Tracker (Wynd Technologies, Inc., CA, USA), and Xiaomi Mi $\mathrm{PM}_{2.5}$ Detector (Beijing Ji Mi Electronics Technology Co., Ltd., China) (Sousan et al., 2017; MorenoRangel et al., 2018; Singer and Delp, 2018).

Although low-cost PM monitors have been well characterized, the studies reflect inherent limitations. First, there is no specified boundary between low-cost PM monitors and low-cost PM sensors. Occasionally, low-cost PM monitors are used as reference instruments to calibrate low-cost PM sensors. At other times, they are treated the same as low-cost PM sensors. It is necessary to distinguish low-cost PM monitors from low-cost PM sensors according to the differences mentioned above. However, it is still uncertain whether they are qualified enough as a reference instrument. Second, calibration methods for low-cost PM monitors are very likely different, but differences are not highlighted in the literature. For low-cost PM sensors, a user calibration procedure will establish the relationship between the PM concentration and an electrical signal (e.g., current, voltage, or pulse width). Hence, the major concern is whether low-cost PM sensors can correlate well 
with reference instruments. However, for low-cost PM monitors, the correlation is between PM concentrations reported by monitors and the reference instrument, and a larger concern is whether the agreement between monitors and the reference instrument is good enough. Hence, linear or polynomial regression may not be sufficient to demonstrate the accuracy of these low-cost PM monitors. Third, an important function of some residential monitors is indicating air quality through a color change, which is a straightforward display. However, few studies have examined whether a color indicator can convey air quality information accurately and promptly. To bridge the scientific gap, in this study, the performance of nine types of popular lowcost PM monitors was compared, including devices from AirVisual Pro, Alphasense, APT, Awair, Dylos, PurpleAir, Foobot, Wynd, and Xiaomi. One monitor from each brand was tested in the laboratory experiments. These monitors were divided into two groups according to their number of channels and type of metrics. As for reference instruments, GRIMM (11C; GRIMM Technologies, Inc., GA, USA) and TSI SidePak (AM530; TSI Inc., MN, USA) were chosen to evaluate these monitors. A chamber was built to provide an adjustable test environment with approximately well-mixed and evenly distributed PM concentrations, together with humidity and temperature control.

\section{METHODS}

In this study, nine popular low-cost personal PM monitors were compared against two reference instruments. The specifications and metrics of the different monitors were compared, as were their features for convenient application. The procedure to compare monitors with different specifications and metrics will be elaborated in this section. A chamber with temperature and humidity control was designed to provide a well-mixed and evenly distributed flow for calibration experiments. The mixing performance of the chamber was examined at random locations to demonstrate its workability. With the temperature around $23 \pm 0.5^{\circ} \mathrm{C}$ and relative humidity around $50 \pm 4 \%$, three types of aerosols - ARD particles, sea salt particles, and incense particles-were generated from dust dispenser, atomizer, and burning incense to evaluate the performance of different monitors.

\section{Specifications of Low-cost PM Monitors and Reference Instruments}

Table 1 lists the specifications of all low-cost PM monitors tested in this study. Although some of the monitors (for example, the Alphasense and Dylos) have been utilized as reference instruments to calibrate low-cost PM sensors as reported in the literature (Rajasegarar et al., 2014; Prabakar et al., 2015; Gao et al., 2016; Hojaiji et al., 2017), we treated all of them as test units since there are no significant price differences between them and other tested monitors. Monitors were classified into two groups on the basis of their working principle and metrics. The Alphasense, APT (Applied Particle Technology Minima), Dylos, and PurpleAir all use a single-particle counter which measures the size distribution for sorting into multiple bins. When particles pass through the measurement area one at a time, the scattered light generates a pulse that is detected by a photodiode, and then the particle size is classified according to the pulse height. The particle sensing modules of the AirVisual, Awair, Foobot, Wynd, and Xiaomi monitors report an analog measurement of the total mass concentration. Particles that pass through the measurement area at the same time scatter light onto the photodiode and the detected light intensity can be correlated with the PM concentration.

Among the first group, the APT and PurpleAir are equipped with a Plantower single-particle sensing module (Plantower Co., Ltd., Beijing, China). The Alphasense and Dylos have their own custom-designed sensing modules. Due to the differences between modules, the data reporting formats of each monitor are different. The APT and PurpleAir monitors, using the low-cost Plantower sensor, report the size distribution of particles ranging from $0.3-10 \mu \mathrm{m}$ in 6 bins. The Alphasense has a better resolution and reports the sizes ranging from $0.3-38 \mu \mathrm{m}$ in 24 bins. The Dylos has only 2 bins for particles larger than $0.5 \mu \mathrm{m}$ and $2.5 \mu \mathrm{m}$ respectively. To make the Dylos results comparable with other monitors in the first group, the number concentration of the second bin $(>2.5 \mu \mathrm{m})$ was subtracted from that of the first bin $(>0.5 \mu \mathrm{m})$ to represent the number concentration of particles smaller than $2.5 \mu \mathrm{m}$. The Alphasense, APT, and PurpleAir not only report the size distribution in the unit of number concentration, but also report mass concentrations of $\mathrm{PM}_{1}, \mathrm{PM}_{2.5}$, and $\mathrm{PM}_{10}$.

For data logging, Alphasense and Dylos do not have a wireless module; hence they need to be connected to a computer to display real-time data. The PurpleAir and APT monitors can upload data to a manufacturer-provided webpage through a Wi-Fi module. The Alphasense, APT, and PurpleAir also have internal off-line data logging system that can record the data on a microSD card in case of connection malfunction. The sampling interval of the APT is adjustable, and in our study was set at 1 minute to be consistent with the Dylos and GRIMM. The Alphasense reported data every 1 second, and the data were averaged over 1 minute too. The PurpleAir has a fixed sampling interval of 80 seconds; hence the data were interpolated to get a 1-minute sampling interval.

The monitors in the second group are targeted for residential use; hence they are cheaper and smaller, with an attractive appearance and a straightforward display. Apart from PM mass concentration, the AirVisual, Awair, and Foobot also monitor the $\mathrm{CO}_{2}$ or VOC concentrations for a more comprehensive air quality measurement. Since they are designed for residential use, important features, for example, the sampling interval or working principle, are not explained thoroughly in the manufacturer's descriptions. For data logging, all five monitors have a wireless module, a Bluetooth chip for the Wynd and a Wi-Fi chip for the rest of the monitors, to synchronize the data to a tablet or phone applications. For the AirVisual and Foobot, after data is synchronized with the application, it can be accessed online with a 10-minute and a 5-minute interval respectively. However, for other monitors, historical data is not accessible 


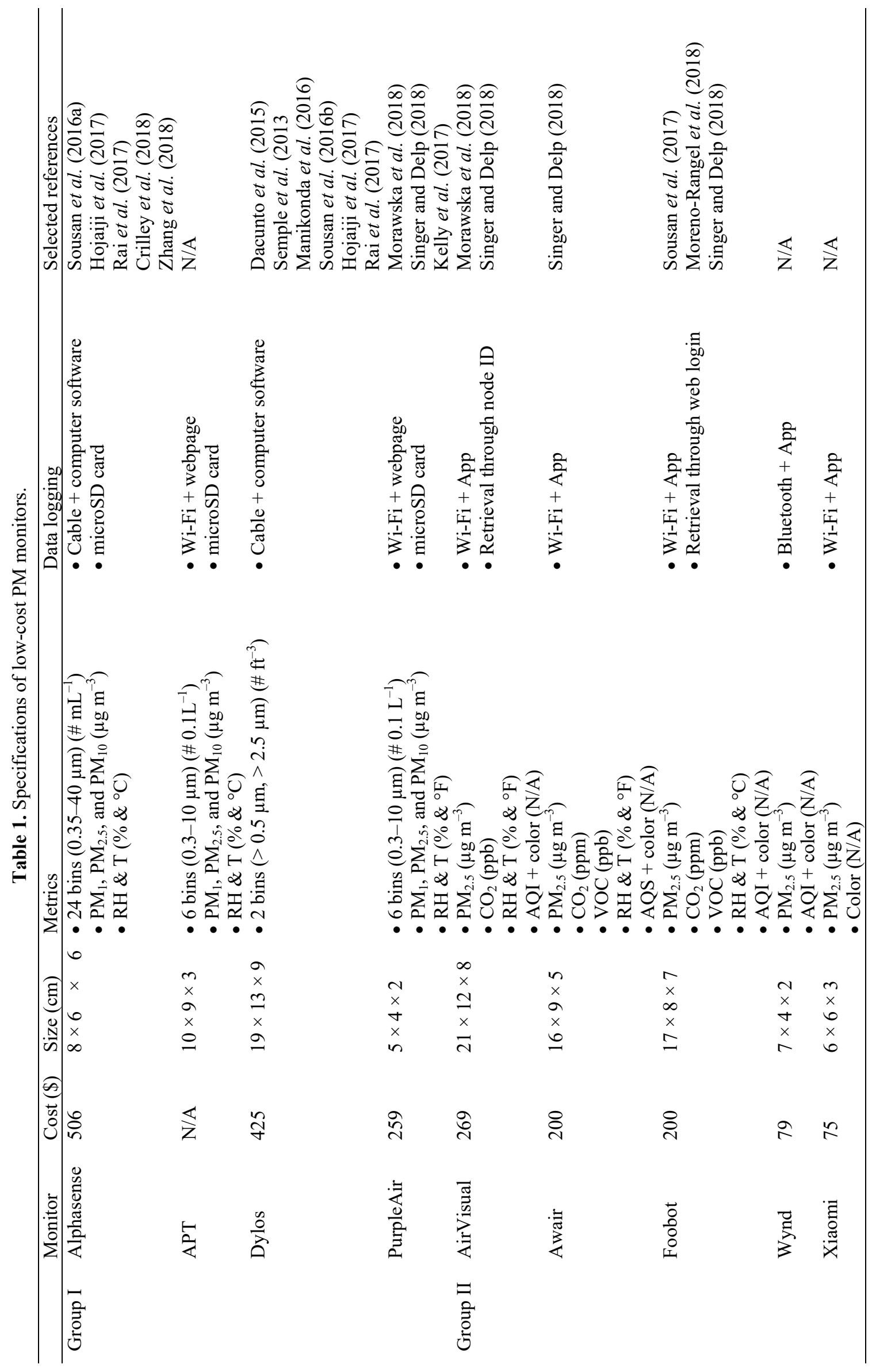


since it is not saved. Our study circumvented this problem by video recording the screen with the app running to record the data and time. The data were manually extracted and averaged over 1-minute intervals. Apart from measuring PM concentrations, these monitors also report an air quality-related index and use different colors to display the air quality more straightforwardly.

Comparing different data logging methods of all tested low-cost PM monitors, a Wi-Fi module or a Bluetooth module can synchronize the data remotely and conveniently as opposed to connections with cables. Generally, monitors with a Bluetooth module can be configured more easily than monitors with a Wi-Fi module, since the Wi-Fi module usually requires a specific type of wireless internet. The Bluetooth module can be connected to user applications straightforwardly; however, the data transfer relies on the user-end application and is restricted in a confined space. On the other hand, monitors with the Wi-Fi module can continue collecting the data even without running user-end applications, which allows long-term and remote data collection. The transferred data can then be accessed through user-end applications (AirVisual, Awair, Foobot, Wynd, and Xiaomi), through web portals (AirVisual, APT, Foobot, and PurpleAir), or through computer software (Alphasense and Dylos).

To compare monitors with different working principles, GRIMM and SidePak were chosen as reference instruments. SidePak reported the $\mathrm{PM}_{2.5}$ mass concentration, which can be correlated directly with different monitors. GRIMM, as a Federal Reference Method (FRM), is a single-particle counter that measures the sizes of PM ranging from 0.25 $32 \mu \mathrm{m}$ and reports the distribution in 31 bins. A bin-wise comparison, as shown in Fig. 1, was conducted for the monitors of the first group to demonstrate the accuracy of their size distribution measurements. Compared to APT, Dylos, and PurpleAir, the Alphasense has more bins and a different distribution; therefore, common ranges for the Alphasense and GRIMM were selected for bin-wise calibration. The APT, Dylos, and PurpleAir use fewer bins than the GRIMM; hence several GRIMM bins were summed to enable comparison.

\section{Air Quality-related Index and Color Display}

The second group of monitors, the AirVisual, Awair, Foobot, Wynd, and Xiaomi, used both numeric indexes and color display to report the air quality. Foobot gives a simplified numeric index on a scale of 0 to 100 indicating air quality ranging from healthy to poor. The Awair presents an air quality score (AQS), and AirVisual and Wynd present the air quality index (AQI). The AQS is scaled from 0 to 100 to indicate the air quality from poor to healthy. However, neither the product manual nor the literature describes how the AQS is calculated. Compared to the AQS, the AQI is a more common parameter for presenting the potential airborne hazards. The component species and calculation details of the AQI vary with local regulations,

\section{Bin Classification}



Fig. 1. Bin classification for monitors in the first group (Alphasense, APT, Dylos, and PurpleAir) in bin-wise comparison against the GRIMM. The dash-dot line and dashed line represent the bin distribution of each monitor and the GRIMM respectively. The $31^{\text {st }}$ bin of GRIMM $(>32 \mu \mathrm{m})$ is not depicted in this figure. The thick box represents the common range of combined bins for comparison. 
but normally, several common air pollutants are included: PM, ozone, VOC, carbon monoxide, and sulfur dioxide (Gao et al., 2015; Hu et al., 2015; U.S. EPA, 2016). A high AQI indicates an unhealthy environment, and the AQI range from low to high is divided into 6 segments with numeric index ranging from 0 to 500 that describes the overall air quality, from excellent to heavily polluted. Since PM is the major object of this study, the AQI mentioned in the following section is calculated based on the PM mass concentration, following the U.S. EPA regulations published in 2016 (U.S. EPA, 2016).

Compared to numeric indexes, color is a more straightforward way of showing air quality. The U.S. EPA divides the AQI into 6 sections, and each section has a signal color (green indicates "good," yellow is "moderate," orange means "unhealthy for sensitive groups," red represents "unhealthy," purple indicates "very unhealthy," and maroon stands for "hazardous"). Ideally, all monitors would use the same color scheme of the AQI, which would make the results directly comparable. In reality, only the AirVisual follows the AQI color distribution. The Awair, Foobot, Wynd, and Xiaomi monitors assign colors according to their own schemes, as shown in Fig. 2. For example, the Foobot uses just 2 colors, blue and orange, and Xiaomi monitor has only 3 colors, green, yellow, and red. To examine whether the color display properly conveys the air quality message, the U.S. EPA regulated AQI was first calculated based on the PM concentration reported by the GRIMM, and the AQI-designated color was used to represent the air quality. The colors reported by the various monitors were recorded at the same time for comparison. Ideally, all the colors would be both identical and accurate representations of the GRIMM-reported PM concentration.

\section{Test Chamber and Aerosol Sources}

A chamber was designed according to Fig. 3(a) to provide a well-mixed and evenly distributed PM flow as the test environment. The aerosol was introduced to the chamber through the inlet on the top and then ventilated through the outlet on the bottom. The exhaust air was filtered by a
HEPA filter, and a flow controlled vacuum pump was used to adjust the flow rate $\left(2-15 \mathrm{~L} \mathrm{~min}^{-1}\right)$ and to control the PM concentration level inside. The interior included a mixing area and a test area, separated by two baffles with a matrix of 1-inch holes. In the mixing area, two fans were used to improve air circulation. A humidifier and a cartridge heater in the mixing area were connected to a humidity sensor and a temperature sensor in the test area to maintain the temperature and humidity in the test area at $23 \pm 0.5^{\circ} \mathrm{C}$ and $50 \pm 4 \%$ respectively. Particles, heat, and humidity were mixed with distorted streamlines. After sufficient mixing, the streamlines passed through two baffles with densely spaced holes to form an evenly distributed laminar flow passing through the test area. During the experiments, monitors of the same group and the SidePak were placed on the lower-level baffle, and the GRIMM was placed outside the chamber but connected to the test area. The monitors of the first group (Alphasense, APT, Dylos, and PurpleAir) and the SidePak were placed on the lower-level baffle as shown in Fig. 3(b). The monitors of the second group (AirVisual, Awair, Foobot, Wynd, and Xiaomi) and the SidePak were placed on the lower-level baffle as shown in Fig. 3(c). To avoid the edge effect, all monitors were placed within $20 \mathrm{~cm}$ from the center. Monitors in Figs. 3(b) and 3(c) were arranged approximately the same distance from the center, and their inlets were directed toward the center.

Incense particles were generated by burning a cone stick incense (Surya Devya Enterprises) near the inlet, and they enter the chamber with the inlet flow. Sea salt particles were generated by atomizing sea salt solutions (sea salts (S9883-1KG; Sigma-Aldrich ${ }^{\circledR}$, MO, USA) and deionized water) with an aerosol generator (Model 3076; TSI Inc., MN, USA). For Arizona Road Dust particles, commercial powders (A1 Ultrafine Test Dust; Powder Technology Inc., MN, USA) were suspended by a fluidized-bed aerosol generator (Model 3400A; TSI Inc., MN, USA). A T-connector was used to connect the aerosol generator with the chamber inlet, and one port of the T-connector was left open to balance the flow rate. The normalized mass distribution of

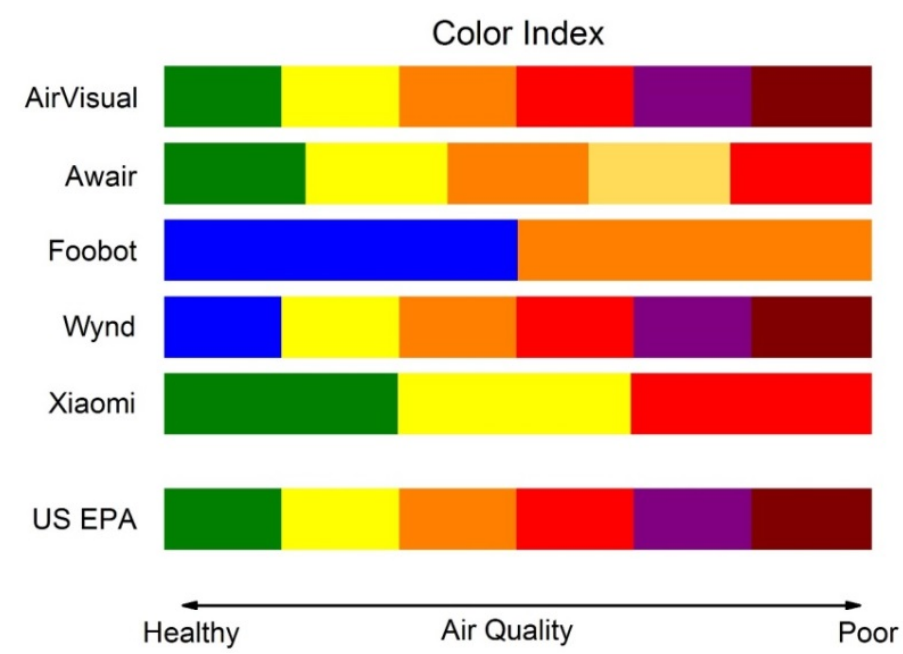

Fig. 2. Color display pattern of each monitor and U.S. EPA stipulated display. 


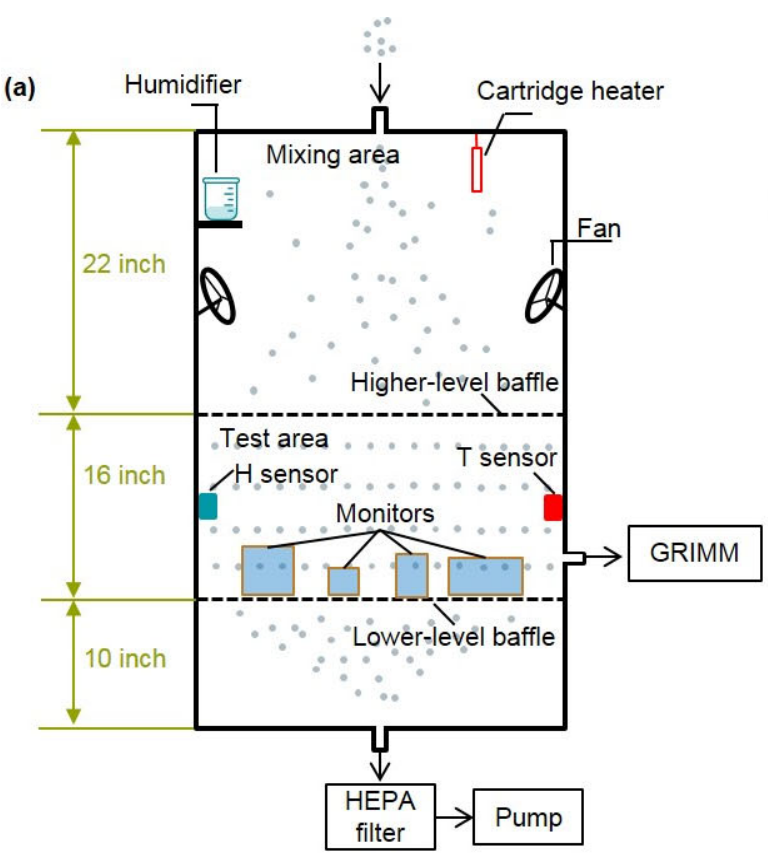

(b)

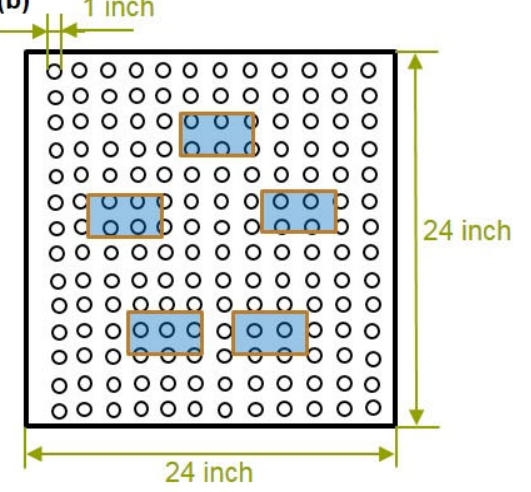

(c)

Fig. 3. (a) The schematic diagram of the chamber used in this study for testing monitors. The chamber provided a wellmixed and evenly distributed PM flow. (b) The monitors of the first group (Alphasense, APT, Dylos, and PurpleAir) and the SidePak were placed on the lower-level baffle as shown. (c) The monitors of the second group (AirVisual, Awair, Foobot, Wynd, and Xiaomi) and the SidePak were placed on the lower-level baffle as shown.

each source was reported by GRIMM, as shown in Fig. 4. Incense particles and sea salt particles peaked around 0.5 and $0.3 \mu \mathrm{m}$ respectively. ARD particles were larger and peaked around 2-4 $\mu \mathrm{m}$. In experiments, the PM concentration was allowed to increase in the chamber after which the PM source was removed. The monitors were tested during the decay of the PM concentration. The space occupied by the monitors and the reference instrument was much smaller than the volume of the chamber $(453 \mathrm{~L})$, and thus no disturbance in the PM levels is expected.

Using incense particles, the uniformity of the PM distribution in the test area was examined by the SidePak and the APT monitor. While the spatial distribution could not be measured by the APT and SidePak monitors due to a time dependent PM profile, they provided similar responses. Thus, the assumption of spatial uniformity of PM distribution is reasonable. To validate this assumption, the response from the APT monitor was correlated with that from the SidePak via a linear regression under $500 \mu \mathrm{g} \mathrm{m}^{-3}$ concentration level. The whole procedure was repeated three times by placing the SidePak monitor and the APT monitor at different locations surrounding the center of the low-level baffle at an approximately similar distance. In all three tests, the response from the APT monitor correlated well with SidePak, and the slopes from different tests were approximately similar $0.847\left(\mathrm{R}^{2}=0.985\right), 0.867\left(\mathrm{R}^{2}=0.984\right)$, and $0.897\left(\mathrm{R}^{2}=0.986\right)$. The average and the standard deviation of the slopes from all three tests were 0.870 and 0.0252 respectively. Such a small standard deviation indicated that the PM was approximately well mixed and evenly distributed in the test area.

\section{Test Agreement between Monitors and Reference Instruments}

Conventional methods usually use the correlation coefficient $\left(\mathrm{R}^{2}\right)$ to evaluate the performance of low-cost PM sensors. $\mathrm{R}^{2}$ values can demonstrate the linearity between monitors and reference instruments; however, they cannot demonstrate the agreement between instruments. Bland and Altman (1986) have demonstrated a method to evaluate the agreement between new techniques and established instruments, and this method has been used to examine whether an instrument is a qualified substitute for the reference instrument (Bland and Altman, 1986, 2003; Astrua et al., 2007). The same method was used in this work to examine the performance of SidePak and the first group of monitors (Alphasense, APT, Dylos, and PurpleAir). The GRIMM monitor and ARD particles were selected as the reference instrument and the PM source respectively. The differences between the $\mathrm{PM}_{2.5}$ mass concentrations reported by monitors and GRIMM were plotted against their average to show the agreement between the monitors and the reference instrument before calibration. The average of the differences $(\sigma)$ and the standard deviation of the differences $(S D)$ were calculated for each monitor. A smaller absolute value of $\sigma$ and a narrower boundary (4SD) indicate a better data quality.

To demonstrate the difference in the performance before and after calibration, a similar procedure was repeated for monitors post calibration. All monitors were calibrated according to either linear regression (SidePak, APT, and PurpleAir) or secondary polynomial regression (Alphasense and Dylos) to obtain an $\mathrm{R}^{2}$ value larger than 0.96 . The data reported by monitors from direct measurements were 


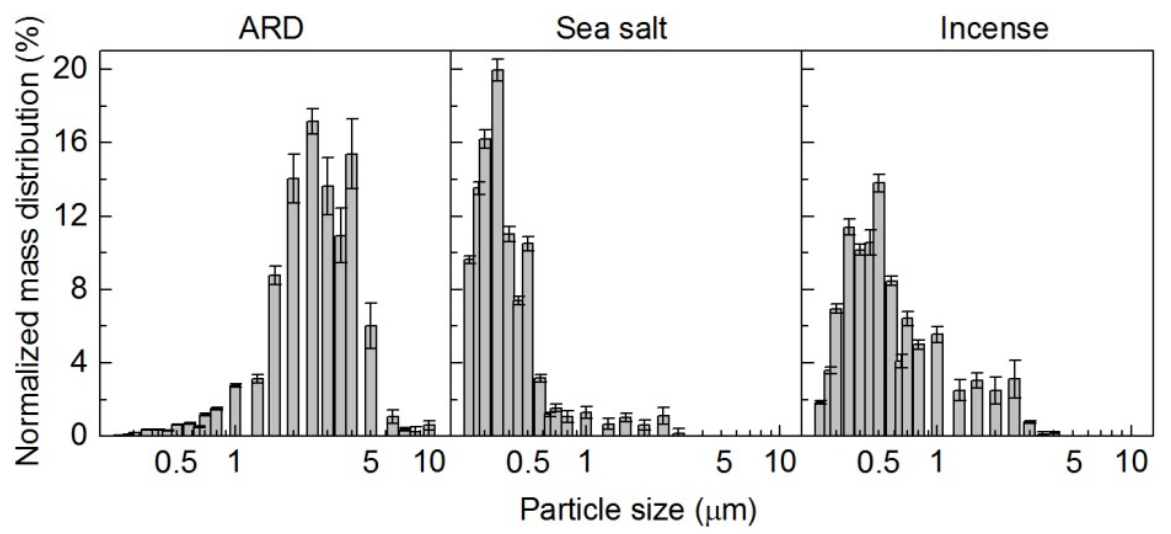

Fig. 4. Normalized mass concentration distributions of ARD particles, sea salt particles, and incense particles generated during the experiments, measured by GRIMM.

re-calculated with calibration equations. After calibration, the differences between the $\mathrm{PM}_{2.5}$ mass concentrations reported by calibrated monitors and GRIMM were plotted against their average, and the $\sigma$ and $S D$ were re-calculated for the post-calibration dataset.

\section{RESULTS AND DISCUSSION}

\section{Alphasense, Dylos, APT, and PurpleAir Monitors \\ Time Response and Correlation Based on PM ${ }_{2.5}$ Mass Concentration}

The $\mathrm{PM}_{2.5}$ mass concentrations reported by monitors and reference instruments for different PM sources are plotted with time in Fig. 5, with a 1-minute sampling interval. The red and blue dashed lines represent the GRIMM and SidePak respectively, and the solid lines with different colors represent different low-cost PM monitors. For ARD particles, the GRIMM and SidePak overlapped well with each other, since they were both calibrated with ARD particles in their factory calibrations. While the GRIMM and SidePak responded simultaneously for incense and sea salt particles, their indicated PM levels were different. Such a difference might be due to the different working principles: The GRIMM is a single-particle counter, but the SidePak performs ensemble measurement. Other monitors also responded simultaneously to PM concentration fluctuations for incense and sea salt particles, although there was a difference among peak values. However, for ARD particles, a noticeable delay was found for the Dylos monitor in repeated tests. Two reasons may lead to such a delay. First, the other monitors directly reported the mass concentration of $\mathrm{PM}_{2.5}$, but for the Dylos monitor, the large bin $(>2.5 \mu \mathrm{m})$ was subtracted from the small bin $(>0.5 \mu \mathrm{m})$ to calculate the number concentration of particles from $0.5-2.5 \mu \mathrm{m}$. Then, the number concentration was converted to the mass concentration by assuming all these particles were $2.5 \mu \mathrm{m}$, with a density of $1200 \mathrm{~kg} \mathrm{~m}^{-3}$. This method did not consider the particles smaller than $0.5 \mu \mathrm{m}$ and used the $2.5 \mu \mathrm{m}$ diameter to represent all particles between 0.5 $2.5 \mu \mathrm{m}$, which might introduce errors into the results, leading to a visual delay. Another possible reason is the misclassification of particles ranging from $0.5-2.5 \mu \mathrm{m}$.
Particles in this range were supposed to be classified into the small bin; however, they may have been accidentally classified into the large bin. Misclassification can influence data accuracy, which results in a noticeable delay. According to the number-based size distribution, approximately $30 \%$ of the ARD particles fall in the range of $0.5-2.5 \mu \mathrm{m}$, but only $6 \%$ of incense particles and $1 \%$ of sea salt particles fall in this range. Thus, the delay is noticeable for ARD particles but almost negligible for incense and sea salt particles.

The $\mathrm{PM}_{2.5}$ mass concentration reported by each monitor was also plotted against the reference instruments for pairwise correlation, as shown in Fig. 6. These monitors had been pre-calibrated; hence better accuracy was expected. Apart from a high $\mathrm{R}^{2}$ value, a slope value approximate to 1 was expected to demonstrate the agreement between monitors and reference instruments. A slope larger or smaller than 1 represents a monitor's overestimation or underestimation of the $\mathrm{PM}_{2.5}$ mass concentration compared to the reference instruments, respectively. The APT and PurpleAir demonstrated good linearity for various sources, with all $\mathrm{R}^{2}$ values larger than 0.94 and 0.91 respectively. For the Dylos, linear regression may not be the optimal fitting method. For the Alphasense, the data slopes were stable when compared against the GRIMM for different sources. One thing worth noting is that the linear correlation may not be sufficient to demonstrate agreement between monitors and reference instruments. It cannot demonstrate how data quality has been improved after calibration. Thus, we plotted the data in a different manner, which will be illustrated in a later section.

\section{Bin-wise Evaluation for Size Distribution Measurement}

Based on the bin classification in Fig. 1, we plotted the number concentration reported by the GRIMM and the tested monitors for different bins in Fig. 7. The dashed line in the figure represents the 1:1 ratio, where the monitors and the GRIMM reported the same results. For comparison, figures of the same source were plotted under the same scales, except for the correlation between the Dylos and the GRIMM for incense measurement. The legends of different monitors are displayed on the rightmost position of each row. 
In Fig. 7, Alphasense demonstrates an overestimation for tested sources. One more thing worth noting is that after saturation, the response from Alphasense decreased with increasing PM concentration, especially for ARD and incense particles. Such an inverted U-shape may be caused by the coincidence error that several small particles passed
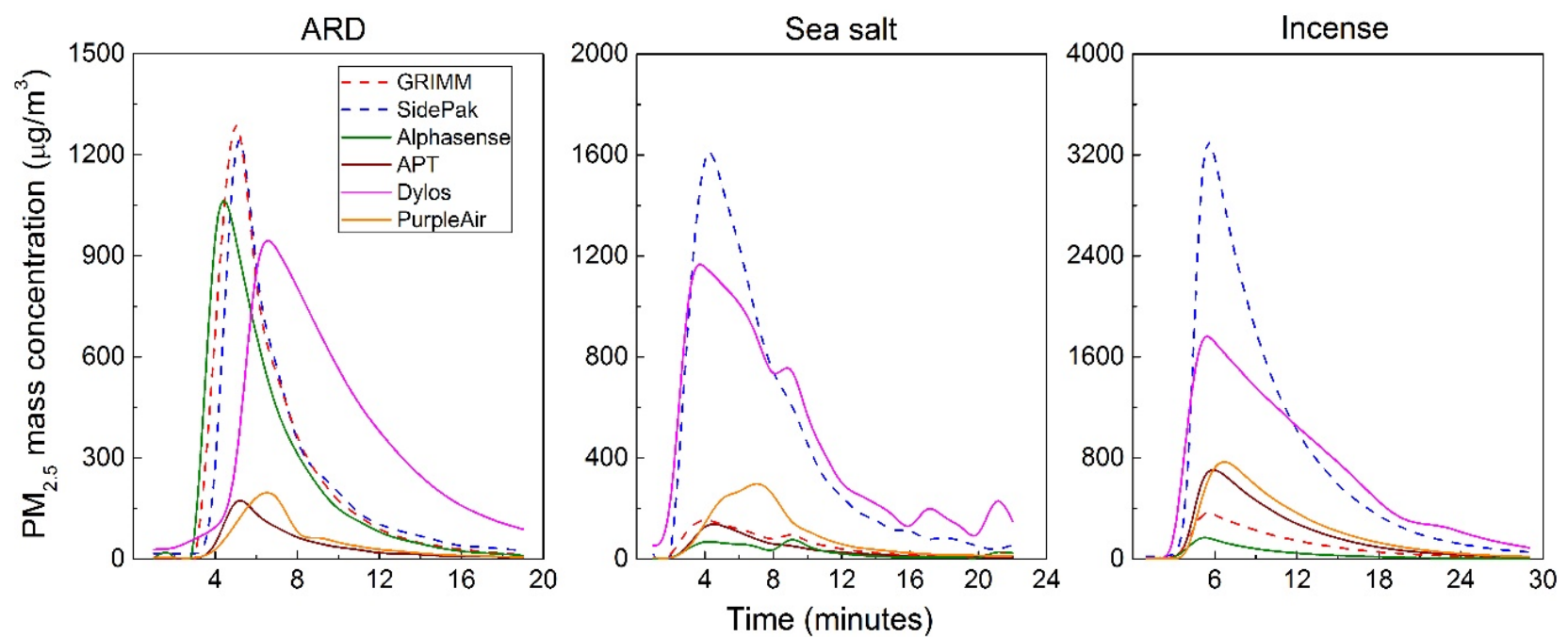

Fig. 5. The $\mathrm{PM}_{2.5}$ mass concentration variation against time for different aerosol sources, reported by reference instruments (GRIMM and SidePak) and monitors (Alphasense, APT, Dylos, and PurpleAir monitors).
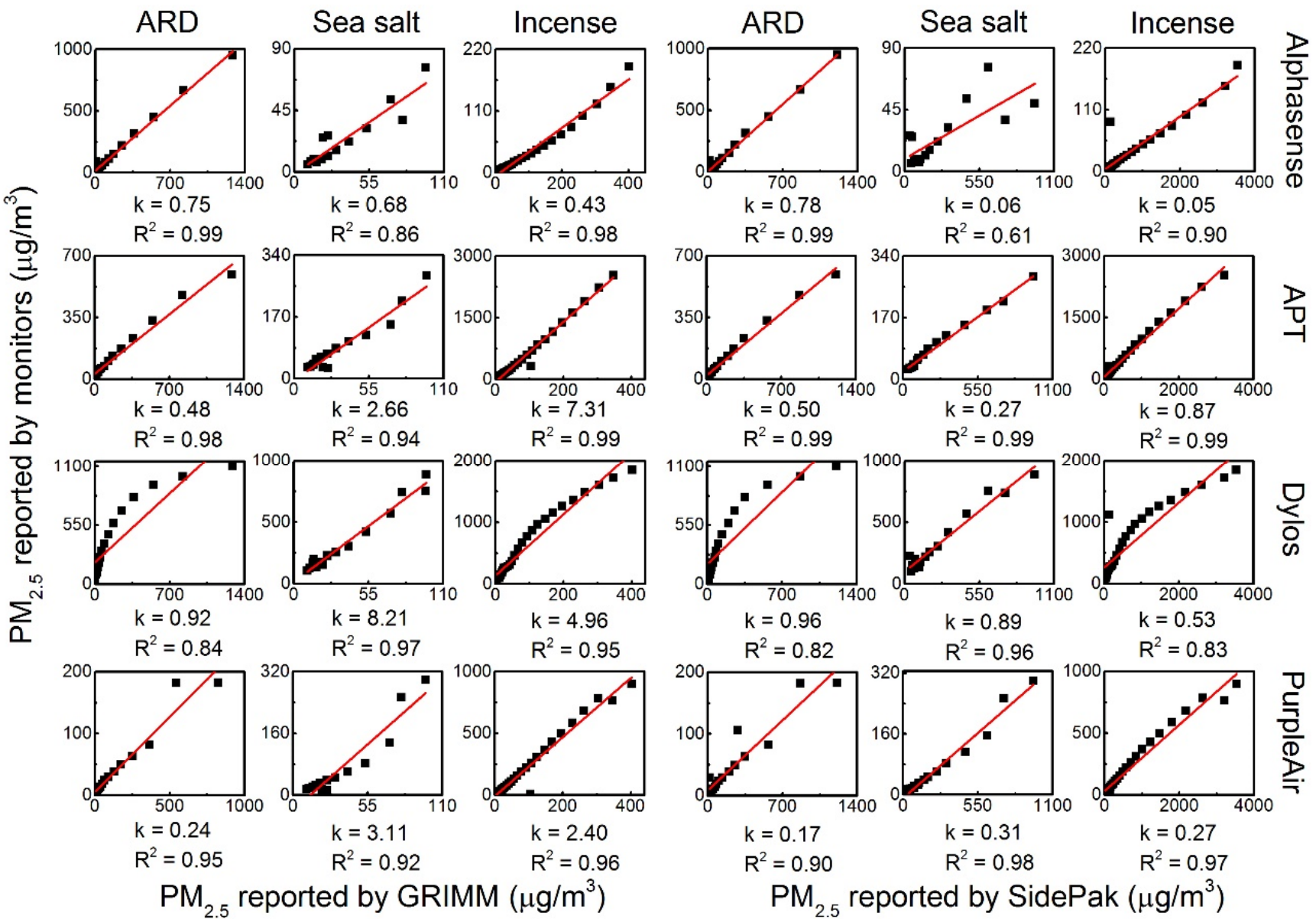

$\mathrm{PM}_{2.5}$ reported by SidePak $\left(\mu \mathrm{g} / \mathrm{m}^{3}\right)$

Fig. 6. Pairwise correlation among the monitors (Alphasense, APT, Dylos, and PurpleAir) and the reference instruments (GRIMM and SidePak) for ARD, sea salt, and incense particles. Slope and $\mathrm{R}^{2}$ values were calculated by least squares regression. 


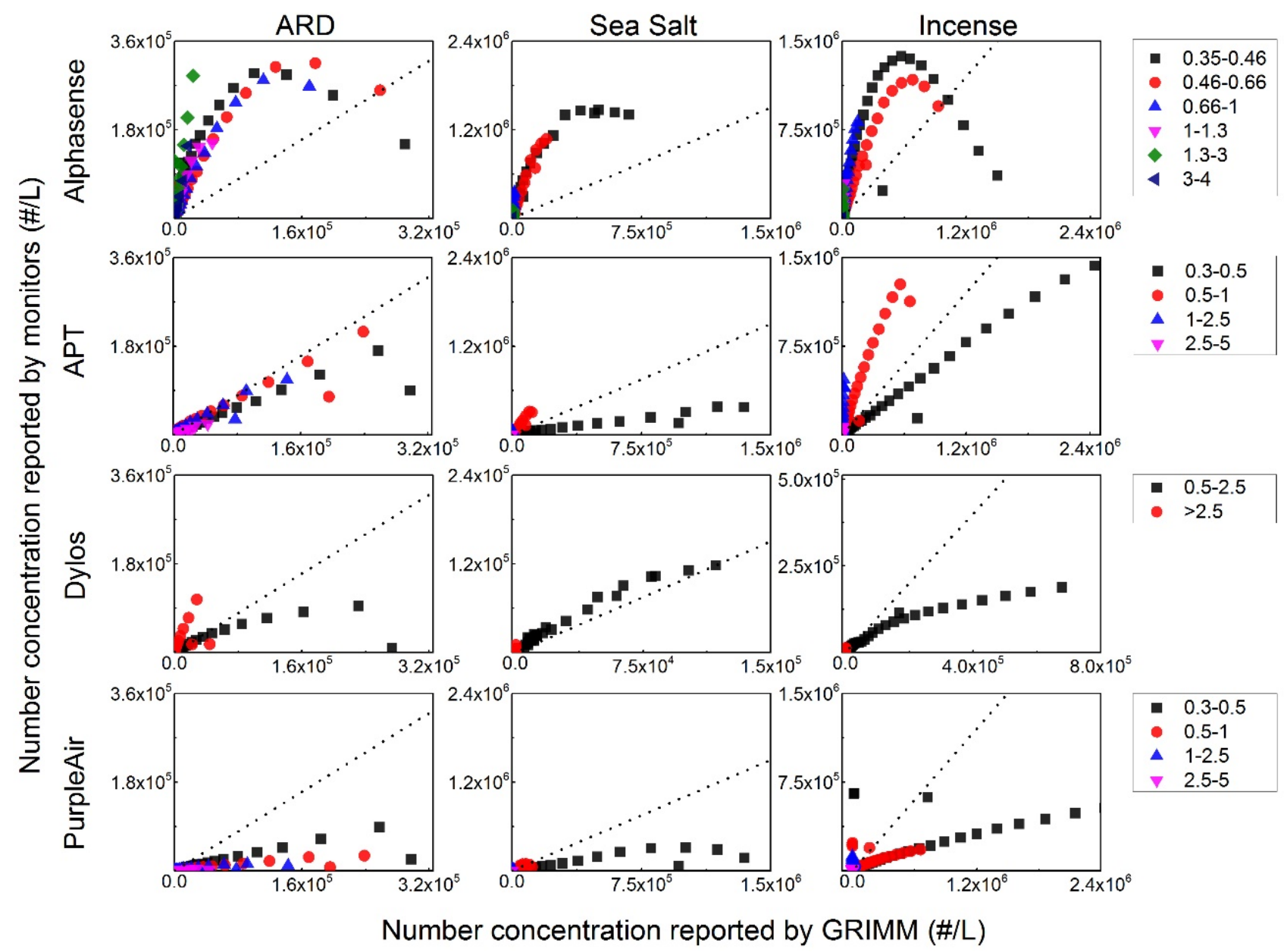

Fig. 7. Bin-wise number concentration comparison of monitors (Alphasense, APT, Dylos, and PurpleAir) and the reference instrument (GRIMM) for different sources in different concentration ranges.

the measuring point at the same time and were characterized as a larger particle by mistake. Coincidence error may lead to an inaccurate size distribution due to the underestimation and overestimation for small and large particles respectively. However, the PM mass correlation in Fig. 6 was not largely influenced by such coincidence error since the misclassified large particles made up the mass loss of small particles. The APT monitor showed a very close estimation for ARD particles; however, for sea salt and incense particles, there was an underestimation and overestimation for $0.3-0.5 \mu \mathrm{m}$ and $0.5-1 \mu \mathrm{m}$ particles respectively. Dylos predicted a very close estimation for sea salt particles; however, it underestimated ARD and incense particles for smaller bins, and overestimated ARD particles for larger bins. PurpleAir monitor demonstrated an underestimation for tested sources.

Fig. 7 also gives a clue on the question whether the calibration procedures should be the same for the optical particle counters and the monitors performing ensemble measurement. For sensors performing ensemble measurement, calibration procedures established the oneto-one relationship between the sensor output and the PM concentration level. However, for optical particle counters, all different channels together contribute to the final mass concentration estimation, and the one-to-one correlation may oversimplify the situation. In Fig. 7, most of the data points could not fall in a narrow range, indicating that no unique calibration factors could be applied for all different channels. Instead, different calibration factors from bin-wise size distribution calibration may produce better accuracy. However, a bin-wise calibration may lower the resolution since common ranges between the monitors and the reference instruments need to be selected. In addition, the bin-wise calibration may be influenced by the PM composition too, since the composition will influence the size characterization, which makes the situation more complicated.

To demonstrate a more straightforward comparison, the size distribution reported by different monitors and the GRIMM is displayed in Fig. 8. Three samples under an approximately stable and high PM concentration were selected for different sources, and the number concentrations reported by the GRIMM and the tested monitors were plotted in shaded and yellow columns, respectively, on the left $y$-axis. The bin ratio, which is the ratio of the number concentration reported by the monitors to that reported by the GRIMM, is plotted in a line to be read from the right y-axis. The dashed line in the figure represents the bin ratio of 1 , where the monitors and the GRIMM reported the same 


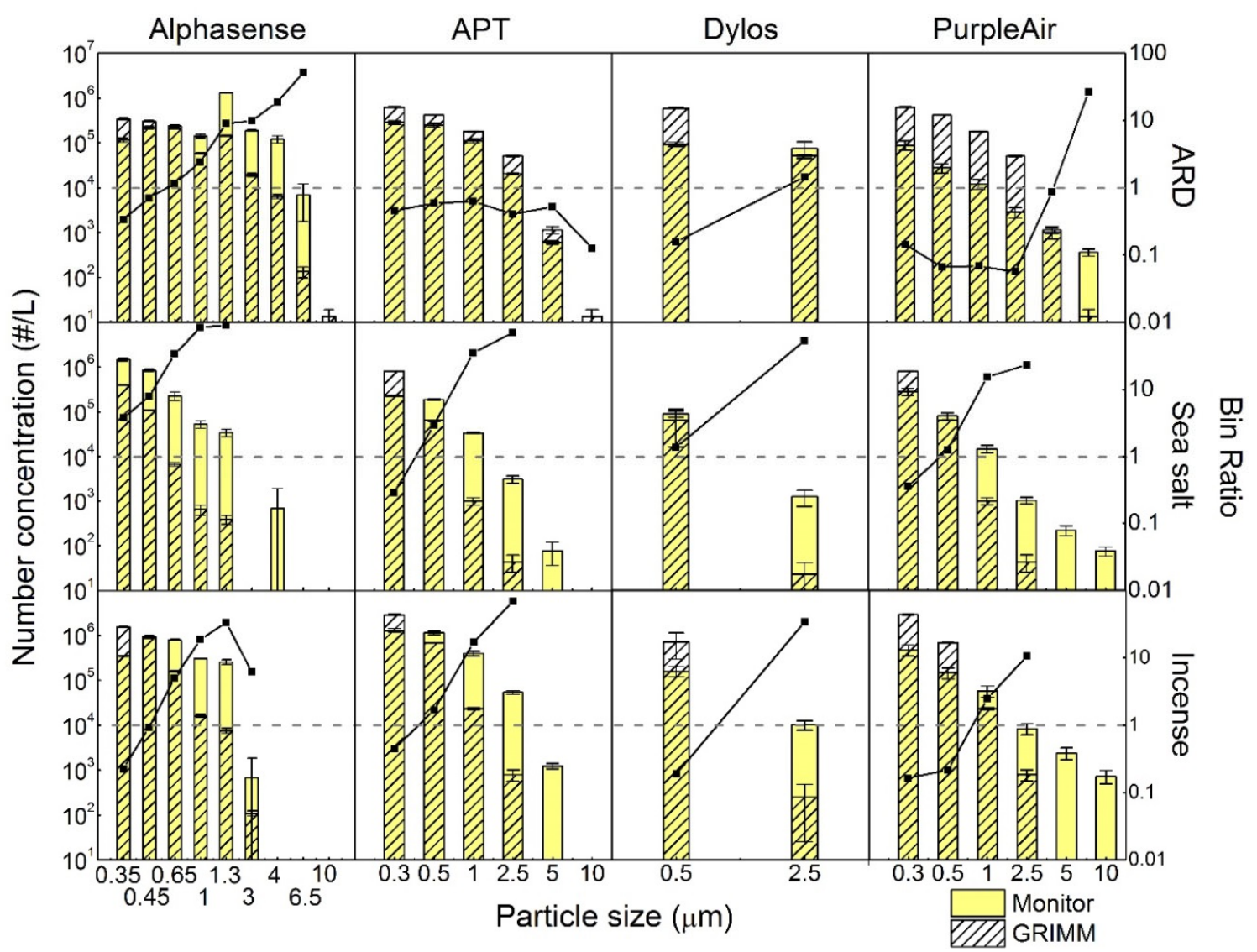

Fig. 8. Size distributions reported by monitors (Alphasense, APT, Dylos, and PurpleAir) and the reference instrument (GRIMM). The number concentrations reported by the monitors and GRIMM are plotted on the left y-axis in yellow and shaded columns respectively. The bin ratio can be read on the right y-axis.

results. Fig. 8 also indicates that no unique calibration factor can be deployed for all channels, although all sensors predicted a roughly correct size distribution. Alphasense and PurpleAir displayed an underestimation and overestimation for small and large particles respectively, which might be due to the coincidence error. The Dylos has only 2 bins; therefore, it is difficult to compare the size distribution. One thing worth noting is that the noticeable overestimation for larger particles might not be adequate to assess the monitors' true performance. The number concentration is very low for larger particles, which may skew the evaluation. The GRIMM detected almost no particles larger than $4 \mu \mathrm{m}$, but the PurpleAir, APT, and Alphasense monitors reported the existence of $10-\mu \mathrm{m}$ particles.

\section{Agreement Before and After Calibration}

As mentioned in the introduction, a large selling point of these low-cost PM monitors is that they arrive assembled and calibrated for deployment. However, according to the results of the pairwise correlation based on the $\mathrm{PM}_{2.5}$ mass concentration and the bin-wise comparison based on the number concentration, discrepancies existed between measurements. Two reasons can explain such discrepancy: different inherent working principles of these monitors, and errors that can be eliminated by calibrations. In other words, the calibration discrepancy is a systematic error, representing a constant drift that can be corrected. However, the inherent discrepancy cannot be eliminated through further calibrations, similar to random errors that cannot be ruled out. To demonstrate the agreement between monitors and the reference instrument, together with distinguishing whether the discrepancies were inherent or the result of inadequate calibration, the ARD $\mathrm{PM}_{2.5}$ data in Fig. 6 was re-plotted as shown in Fig. 9 with the method reported by Bland and Altman et al. (1986). On each figure, the dashed line in the middle is the mean of differences $(\sigma)$, and the dotted lines $(\sigma \pm 2 S D)$ indicate the upper and lower boundaries of the differences.

Before calibration, the low-cost PM monitors exhibited a strong relationship between the difference and the average. For the Alphasense, APT, and PurpleAir, the difference increased negatively with the average, which illustrated that the calibration factor provided by the manufacturer was too large. Therefore, these monitors overestimated the $\mathrm{PM}_{2.5}$ mass concentration compared to the GRIMM. The overestimation was proportional to the measurement scale; hence the difference and the average demonstrated a strong correlation. The Dylos showed a positive correlation between the difference and average, which may be caused by too small a calibration factor. The response of these lightscattering monitors depends on the type of PM sources used, and this has been demonstrated in previous studies 


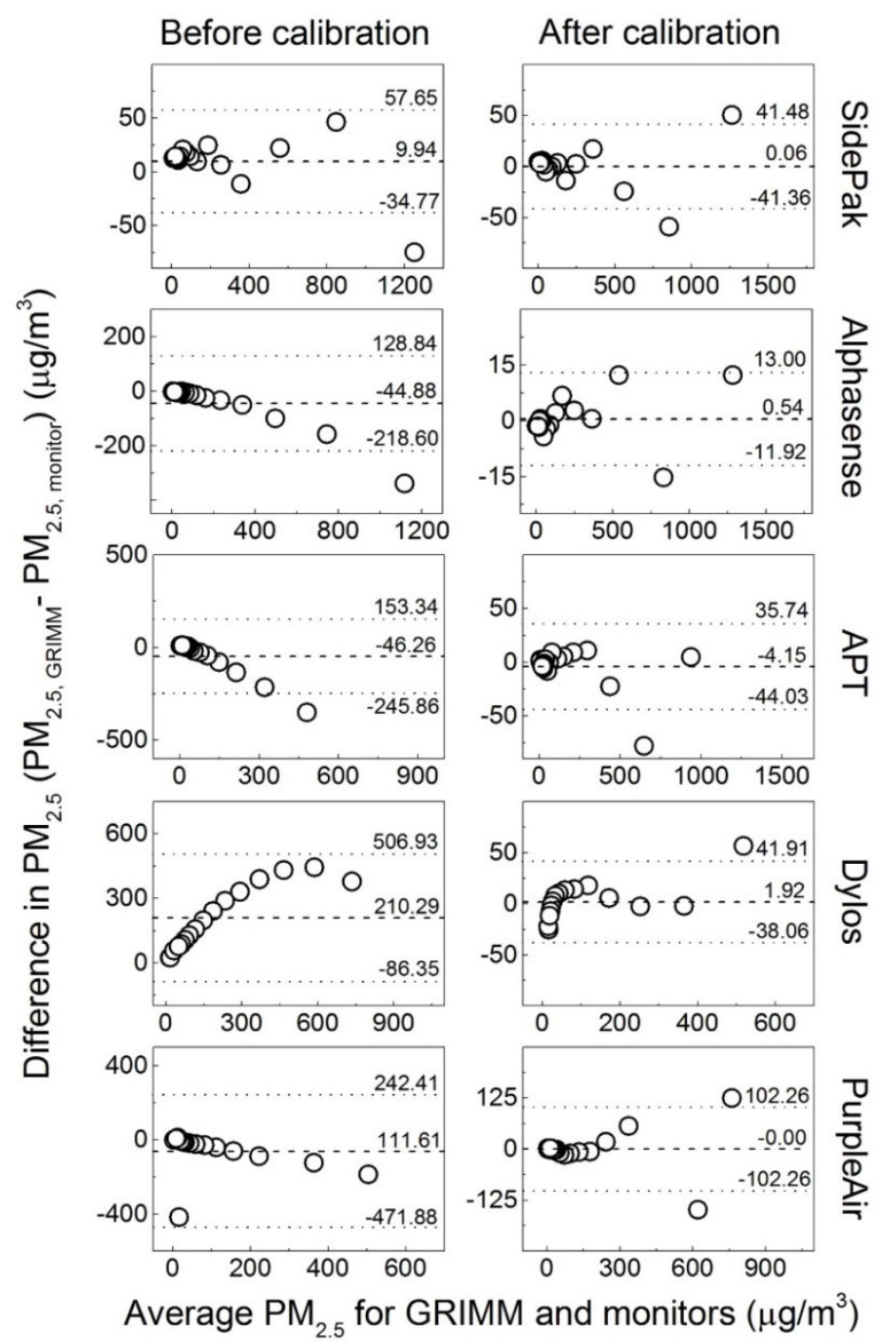

Fig. 9. Differences and averages of $\mathrm{PM}_{2.5}$ mass concentrations reported by the monitors and GRIMM for examining the agreement before and after calibration.

(Jiang et al., 2011; Li and Biswas, 2017). Therefore, if monitors were calibrated based on different types of PM sources other than ARD particles, they may demonstrate a disagreement in user-defined calibration using ARD particles. For all four monitors, the gaps between boundaries ( $4 S D$ ) had shrunk observably and the $\sigma$ had been reset closer to zero after applying calibration equations, which demonstrate that calibration deviations for these four monitors were corrected after user calibration.

One thing worth noting is that whether before or after calibration, the SidePak did not show a strong correlation between the difference and the average. It showed the narrowest gap between boundaries $(4 S D)$ before calibration when compared to other monitors. After calibration, the width of the gap $(4 S D)$ remained the same, and the mean of the difference $(\sigma)$ was reset closer to zero. This behavior illustrated that user calibration did not significantly improve the data quality of the SidePak. Therefore, user calibration is not necessary for SidePak in measuring ARD $\mathrm{PM}_{2.5}$ mass concentration.
In summary, the factory calibration is sufficient for the SidePak for measuring ARD $\mathrm{PM}_{2.5}$; however, the user calibration improves the data quality of the other four monitors. After the user calibration, the distribution of differences demonstrated a narrower difference boundary $(4 S D)$ and a smaller absolute value of $\sigma$, which supports the elimination of calibration deviation. After user calibration, the Alphasense demonstrated the best performance, with an even narrower gap of boundaries (4SD) than the SidePak. The reason might be that both the GRIMM and the Alphasense use a single-particle counter, but the SidePak uses ensemble measurement. Hence, after the user calibration, the Alphasense demonstrated a greater consistency with the GRIMM data.

\section{AirVisual, Awair, Foobot, Wynd, and Xiaomi Monitors Time Response and Correlation Based on the PM ${ }_{2.5}$ Mass Concentration}

Similar to the first group, the $\mathrm{PM}_{2.5}$ mass concentration reported by different monitors and reference instruments 
were plotted against time. As shown in Fig. 10, the monitors responded almost simultaneously to increasing PM concentrations. However, it is noticeable that the AirVisual demonstrated a different shape due to its 5-minute sampling interval. The Awair and Wynd saturated very quickly in the tests, especially for ARD and incense particles. This saturation can be observed more clearly in Fig. 11, which plots the $\mathrm{PM}_{2.5}$ mass concentration reported by the monitors against the reference instruments.

Fig. 11 shows that all five monitors demonstrated a good linear correlation with the GRIMM or SidePak. The AirVisual had a longer sampling interval, leading to segmented scattered data. The Awair and Wynd showed lower saturation concentrations, and the linear fitting curve in the figure is based on the unsaturated part. The Foobot and Xiaomi correlated well with the GRIMM and SidePak over the full range for different PM sources. However, for AirVisual, Awair, and Wynd, saturation occurred at a lower concentration level. The Foobot presented a high $\mathrm{R}^{2}$ value with a slope close to 1 for ARD particles; hence the Foobot has been calibrated well for ARD particle measurement in the factory setting.

\section{Color Indication}

Unlike the first group of low-cost monitors, the second group of monitors is mainly for residential use. Hence conveying the air quality precisely to users is the first concern, rather than accurately measuring the PM mass concentration. They all convey the air quality through a straightforward color display. Therefore, instead of focusing on the statistics for data quality, we recorded the color change pattern to examine whether each monitor could trigger an air quality alarm promptly. The $\mathrm{PM}_{2.5}$ mass concentration reported by the GRIMM was selected as the reference. The U.S. AQI was calculated based on the reference mass concentration; then color was chosen per the U.S. EPA regulations. The color change of the monitors was recorded from their user applications and then correlated with the $\mathrm{PM}_{2.5}$ mass concentration reported by the GRIMM, as shown in Fig. 12.

Among these monitors, the AirVisual has the same color pattern as the U.S. AQI; the Awair and Wynd each had a similar color pattern. The Awair does not use the color maroon, which represents the poorest air quality in the U.S. AQI. The Wynd uses a blue instead of green for good air quality. The Xiaomi and Foobot have just 3 and 2 colors respectively - a very simplified color scheme that is inconvenient for comparison. For example, for ARD particles, when other monitors turned red or maroon indicating unhealthy air quality, the Xiaomi monitor still displayed green, indicating a healthy environment, which is misleading to users. Among the monitors in the second group, the Foobot most closely predicted the PM mass concentration, but its color pattern is very different from that of the U.S. AQI or other monitors. Nevertheless, for Foobot, the color change between blue and orange always happened when the U.S. AQI was displaying a yellow color, indicating moderate air quality, which can be considered as consistent and accurate. Considering the difficulty of comparing different color patterns, we left the Foobot and Xiaomi out of the comparison and focused only on the color indicating function of the AirVisual, Awair, and Wynd.

Among the AirVisual, Awair, and Wynd, the Wynd triggered the alarm ahead of the U.S. AQI for all three different sources; hence, the Wynd is qualified as a residential monitor for generating prompt alarms. The Awair had an early response to incense particles, almost simultaneous response to ARD particles, and a delayed response to sea salt particles. Therefore, the Awair is more sensitive to combustion particles, and may not be very sensitive to sea salt particles. The AirVisual demonstrated an early alarm for incense and sea salt particles, and a late alarm for ARD particles. However, as mentioned before, the AirVisual measured the PM level and synchronized the data every 5 minutes, which may affect the color display. In general, although each monitor has different responses to various
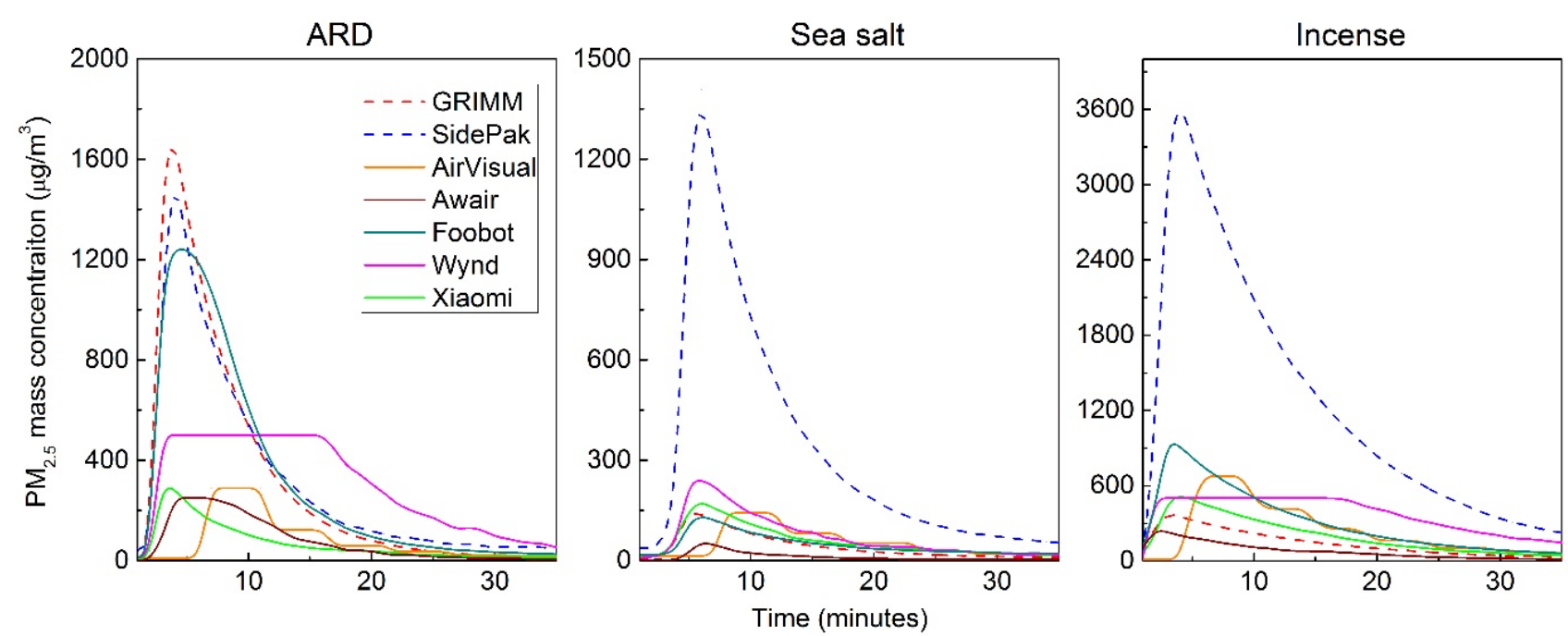

Fig. 10. $\mathrm{PM}_{2.5}$ mass concentration variation against time for different aerosol sources, reported by the reference instruments (GRIMM and SidePak) and monitors (AirVisual, Awair, Foobot, Wynd, and Xiaomi). 


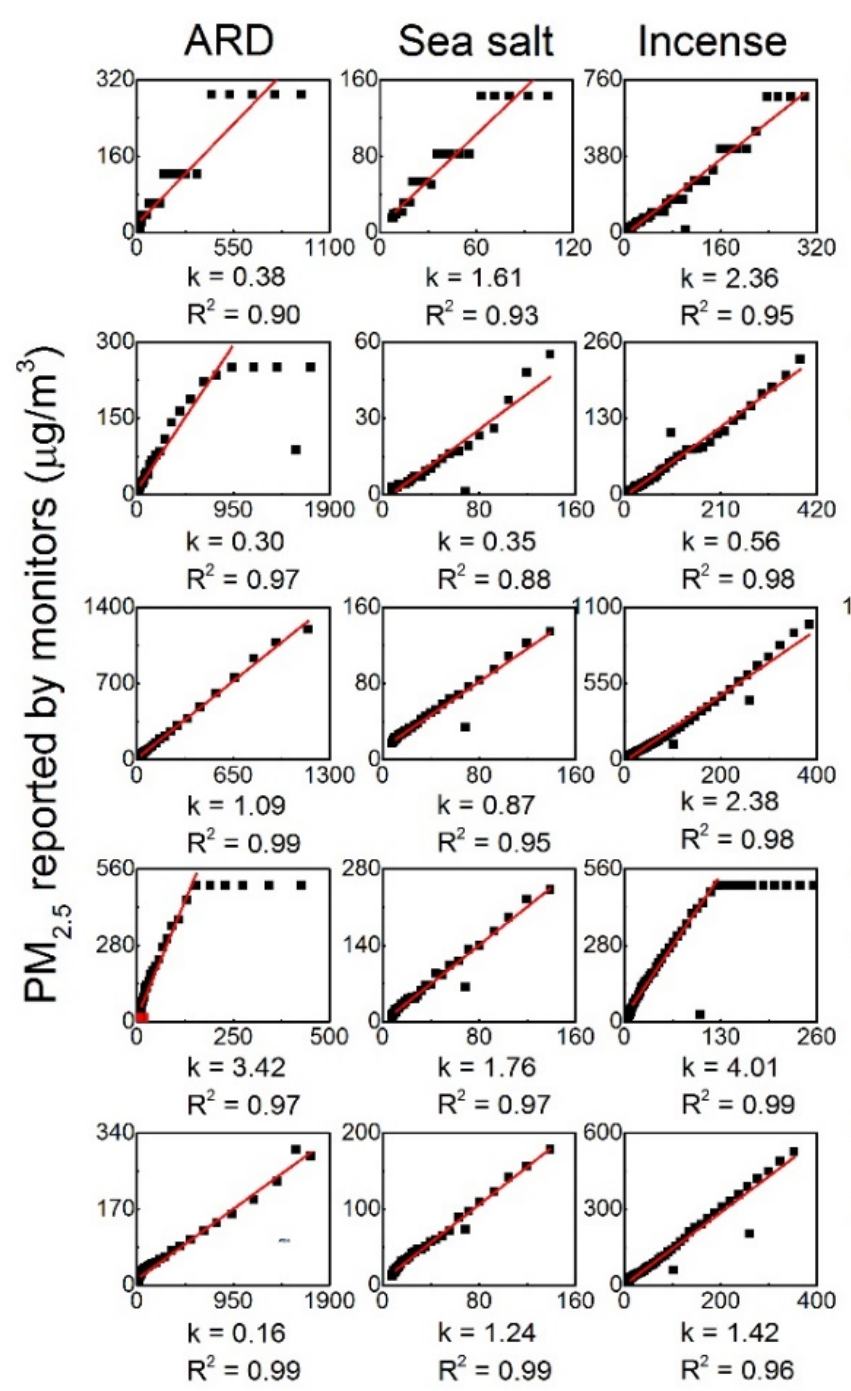

$\mathrm{PM}_{2.5}$ reported by GRIMM $\left(\mu \mathrm{g} / \mathrm{m}^{3}\right)$
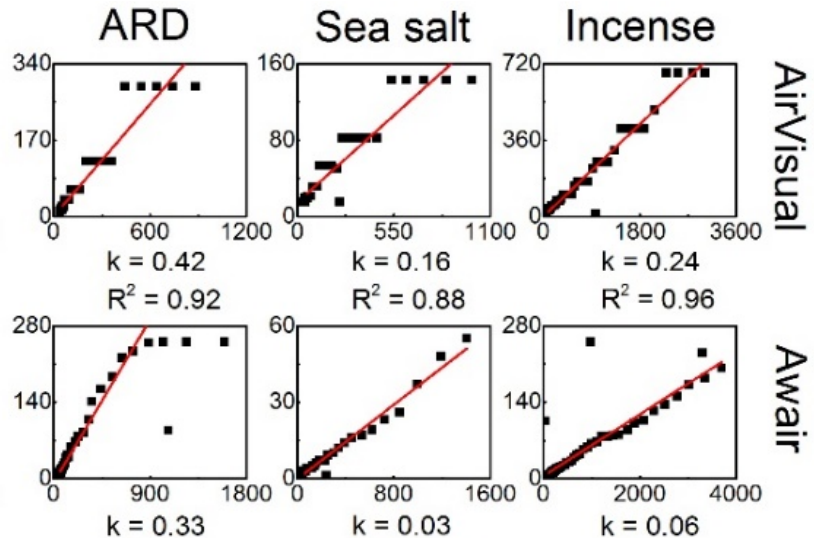

\section{$\stackrel{D}{\stackrel{D}{7}}$}
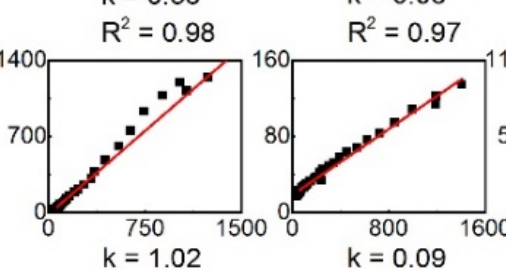

$k=0.06$

$\mathrm{R}^{2}=0.78$
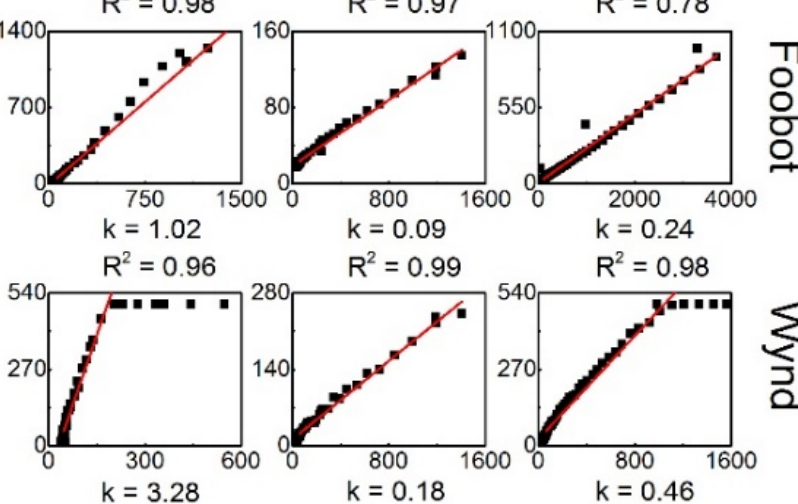

\section{$\sum_{\vdots}$}
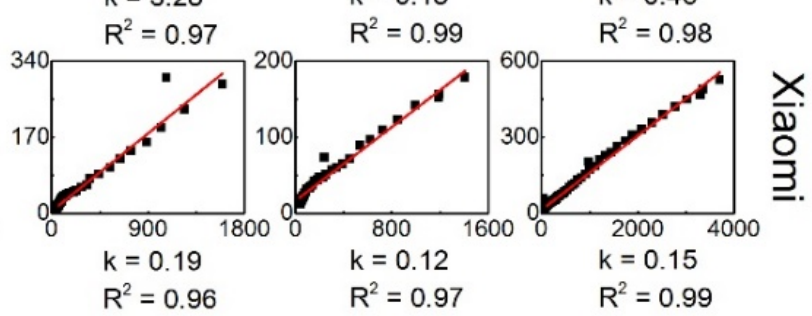

$\mathrm{PM}_{2.5}$ reported by SidePak $\left(\mu \mathrm{g} / \mathrm{m}^{3}\right)$

Fig. 11. Pairwise correlation among the monitors (AirVisual, Awair, Foobot, Wynd, and Xiaomi) and the reference instruments (Grimm and SidePak) for ARD, sea salt, and incense particles. Slope and $\mathrm{R}^{2}$ values were calculated by the least squares regression for the unsaturated range.

sources, the monitors of the second group, apart from the Xiaomi monitor, indicated the air quality moderately well.

\section{CONCLUSIONS}

This study selected nine popular low-cost PM monitors, classified them into two groups based on their features and working principle, and compared their performance within each group. The first group, which comprised the Alphasense, APT, Dylos, and PurpleAir monitors, were evaluated as potential substitutes for the two reference instruments, a GRIMM and a SidePak. Although all four of the tested monitors demonstrated good linearity with the reference instruments, prior to calibration, they showed poor agreement with the latter in measuring ARD particles. One reason may be that these monitors were factorycalibrated using other types of PM, the light scattering signatures of which differ from that of ARD, consequently affecting the measurement. Furthermore, no single calibration factor was able to fit all the channels of a monitor, suggesting that the factor varies depending on the size bin. Thus, data quality can be guaranteed only with user-defined bin-by-bin calibration for the target PM. Coincidence errors, leading to biased size distributions, were observed at high concentrations but were found to have little impact on the reported mass concentrations.

The second group, which comprised the AirVisual, Awair, Foobot, Wynd, and Xiaomi monitors, were examined as reporters of air quality; specifically, we evaluated the efficacy of their color-coded indexes in communicating air quality changes promptly and accurately. All of the monitors demonstrated good linearity with the reference instruments, and four of them (Xiaomi Mi $\mathrm{PM}_{2.5}$ Detector being the exception) displayed the air quality level with moderate accuracy.

Overall, the monitors we tested provide low-cost sensors 

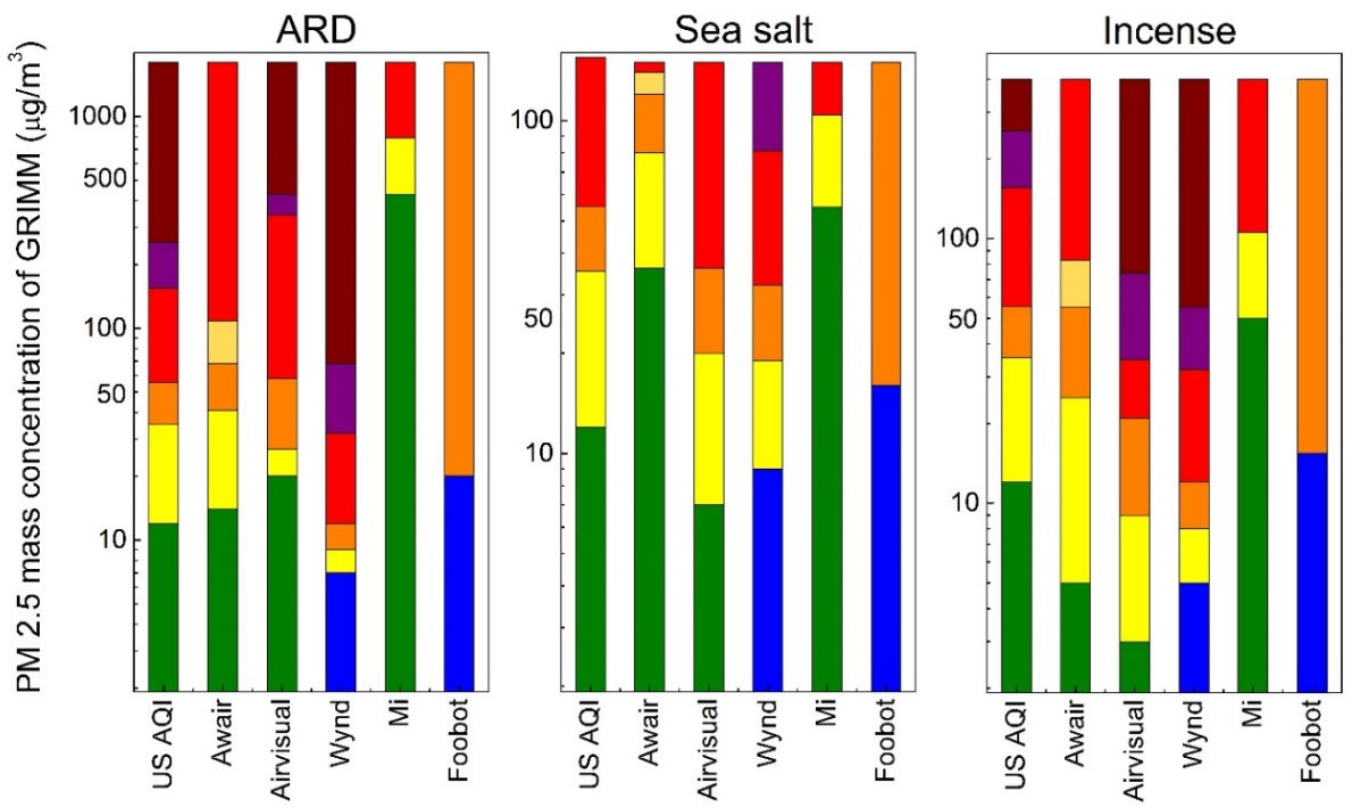

Fig. 12. Color display patterns of the monitors (AirVisual, Awair, Foobot, Wynd, and Xiaomi) for different aerosol sources. The color change for the U.S. AQI column is based on the $\mathrm{PM}_{2.5}$ mass concentration reported by the GRIMM. The color changes of other monitors were recorded from user applications.

in products that are ready for use. Low-cost PM sensors usually exhibit $\mathrm{R}^{2}$ values above 0.8 in laboratory tests, although these values are lower in field tests $(>0.5)$. Excepting the Alphasense OPC-N3's measurement of sea salt particles $\left(\mathrm{R}^{2}=0.61\right)$, the $\mathrm{R}^{2}$ values we obtained with the low-cost monitors following laboratory calibration were larger than 0.82 , which is comparable to results reported in the literature.

The current work could be extended as follows. First, the long-term stability of these low-cost PM monitors-a potential issue with longer field deployment - should be assessed. As an example, Crilley et al. (2018) applied nonparametric ranking to analyze the drift in the response of Alphasense OPC-N2 monitors. Second, multiple units of the same model should be tested to ensure repeatability of performance. Third, field evaluations should be conducted, as monitors may experience complex variations in the target PM during real-world conditions, which may compromise their data quality (Manikonda et al., 2016; Castell et al., 2017; Sayahi et al., 2019).

\section{ACKNOWLEDGEMENTS}

The work was partially supported by a grant from the National Science Foundation (SusChEM: Ultrafine Particle Formation in Advanced Low Carbon Combustion Processes; CBET 1705864). Simar Kaur and Jiayu Li were supported by grants from the McDonnell Academy Global Energy and Environmental Partnership (MAGEEP).

\section{REFERENCES}

Aliyu, Y.A. and Botai, J.O. (2018). Reviewing the local and global implications of air pollution trends in Zaria, northern Nigeria. Urban Clim. 26: 51-59.

Allen, G., Sioutas, C., Koutrakis, P., Reiss, R., Lurmann, F.W. and Roberts, P.T. (1997). Evaluation of the TEOM ${ }^{\circledR}$ method for measurement of ambient particulate mass in urban areas. J. Air Waste Manage. Assoc. 47: 682-689.

Astrua, M., Ichim, D., Pennecchi, F. and Pisani, M. (2007). Statistical techniques for assessing the agreement between two instruments. Metrologia 44: 385.

Bland, J.M. and Altman, D. (1986). Statistical methods for assessing agreement between two methods of clinical measurement. Lancet 327: 307-310.

Bland, J.M. and Altman, D.G. (2003). Applying the right statistics: Analyses of measurement studies. Ultrasound Obstet. Gynecol. 22: 85-93.

Brook, R.D., Rajagopalan, S., Pope, C.A., Brook, J.R., Bhatnagar, A., Diez-Roux, A.V., Holguin, F., Hong, Y., Luepker, R.V. and Mittleman, M.A. (2010). Particulate matter air pollution and cardiovascular disease: An update to the scientific statement from the American Heart Association. Circulation 121: 2331-2378.

Burnett, R.T., Pope III, C.A., Ezzati, M., Olives, C., Lim, S.S., Mehta, S., Shin, H.H., Singh, G., Hubbell, B., Brauer, M. and Anderson, H.R. (2014). An integrated risk function for estimating the global burden of disease attributable to ambient fine particulate matter exposure. Environ. Health Perspect. 122: 397-403.

Castell, N., Dauge, F.R., Schneider, P., Vogt, M., Lerner, U., Fishbain, B., Broday, D. and Bartonova, A. (2017). Can commercial low-cost sensor platforms contribute to air quality monitoring and exposure estimates? Environ. Int. 99: 293-302.

Cohen, A.J., Ross Anderson, H., Ostro, B., Pandey, K.D., Krzyzanowski, M., Künzli, N., Gutschmidt, K., Pope, A., Romieu, I. and Samet, J.M. (2005). The global 
burden of disease due to outdoor air pollution. $J$. Toxicol. Environ. Health, Part A 68: 1301-1307.

Crilley, L.R., Shaw, M., Pound, R., Kramer, L.J., Price, R., Young, S., Lewis, A.C. and Pope, F.D. (2018). Evaluation of a low-cost optical particle counter (Alphasense OPCN2) for ambient air monitoring. Atmos. Meas. Tech. 11: 709-720.

Dacunto, P.J., Klepeis, N.E., Cheng, K.C., AcevedoBolton, V., Jiang, R.T., Repace, J.L., Ott, W.R. and Hildemann, L.M. (2015). Determining $\mathrm{PM}_{2.5}$ calibration curves for a low-cost particle monitor: Common indoor residential aerosols. Environ. Sci. Processes Impacts 17: 1959-1966.

Gao, Q., Liu, J., Li, W. and Gao, W. (2015). Comparative analysis and inspiration of air quality index between China and America. Huan Jing Ke Xue 36: 1141-1147.

Gao, Y., Dong, W., Guo, K., Liu, X., Chen, Y., Liu, X., $\mathrm{Bu}$, J. and Chen, C. (2016). Mosaic: A low-cost mobile sensing system for urban air quality monitoring. IEEE INFOCOM 2016 - The $35^{\text {th }}$ Annual IEEE International Conference on Computer Communications, 2016, San Francisco, CA, USA, pp. 1-9.

Hapidin, D.A., Saputra, C., Maulana, D.S., Munir, M.M. and Khairurrijal, K. (2019). Aerosol chamber characterization for commercial particulate matter (PM) sensor evaluation. Aerosol Air Qual. Res. 19: 181-194.

He, H.D., Li, M., Wang, W.L., Wang, Z.Y. and Xue, Y. (2018). Prediction of $\mathrm{PM}_{2.5}$ concentration based on the similarity in air quality monitoring network. Build. Environ. 137: 11-17.

Hojaiji, H., Kalantarian, H., Bui, A.A.T., King, C.E. and Sarrafzadeh, M. (2017). Temperature and humidity calibration of a low-cost wireless dust sensor for realtime monitoring. 2017 IEEE Sensors Applications Symposium (SAS), 2017, Glassboro, NJ, USA, pp. 1-6.

Holstius, D.M., Pillarisetti, A., Smith, K. and Seto, E. (2014). Field calibrations of a low-cost aerosol sensor at a regulatory monitoring site in california. Atmos. Meas. Tech. 7: 1121-1131.

Hu, J., Ying, Q., Wang, Y. and Zhang, H. (2015). Characterizing multi-pollutant air pollution in China: Comparison of three air quality indices. Environ. Int. 84: 17-25.

Jeon, Y., Cho, C., Seo, J., Kwon, K., Park, H., Oh, S. and Chung, I.J. (2018). IoT-based occupancy detection system in indoor residential environments. Build. Environ. 132: 181-204.

Jiang, R.T., Acevedo-Bolton, V., Cheng, K.C., Klepeis, N.E., Ott, W.R. and Hildemann, L.M. (2011). Determination of response of real-time SidePak AM510 monitor to secondhand smoke, other common indoor aerosols, and outdoor aerosol. J. Environ. Monit. 13: 1695-1702.

Johnson, K.K., Bergin, M.H., Russell, A.G. and Hagler, G.S. (2018). Field test of several low-cost particulate matter sensors in high and low concentration urban environments. Aerosol Air Qual. Res. 18: 565-578.

Kelly, K., Whitaker, J., Petty, A., Widmer, C., Dybwad, A., Sleeth, D., Martin, R. and Butterfield, A. (2017).
Ambient and laboratory evaluation of a low-cost particulate matter sensor. Environ. Pollut. 221: 491-500.

Kim, J.J., Jung, S.K. and Kim, J.T. (2010). Wireless monitoring of indoor air quality by a sensor network. Indoor Built Environ. 19: 145-150.

Kim, J.Y., Chu, C.H. and Shin, S.M. (2014). ISSAQ: An integrated sensing systems for real-time indoor air quality monitoring. IEEE Sens. J. 14: 4230-4244.

Klepeis, N.E., Ott, W.R. and Switzer, P. (2007). Real-time measurement of outdoor tobacco smoke particles. J. Air Waste Manage. Assoc. 57: 522-534.

Kumar, P., Morawska, L., Martani, C., Biskos, G., Neophytou, M., Di Sabatino, S., Bell, M., Norford, L. and Britter, R. (2015). The rise of low-cost sensing for managing air pollution in cities. Environ. Int. 75: 199205.

Leavey, A., Fu, Y., Sha, M., Kutta, A., Lu, C., Wang, W., Drake, B., Chen, Y. and Biswas, P. (2015). Air quality metrics and wireless technology to maximize the energy efficiency of HVAC in a working auditorium. Build. Environ. 85: 287-297.

Li, J. and Biswas, P. (2017). Optical characterization studies of a low-cost particle sensor. Aerosol Air Qual. Res. 17: 1691-1704.

Li, J., Li, H., Ma, Y., Wang, Y., Abokifa, A.A., Lu, C. and Biswas, P. (2018). Spatiotemporal distribution of indoor particulate matter concentration with a low-cost sensor network. Build. Environ. 127: 138-147.

Liu, D., Zhang, Q., Jiang, J. and Chen, D.R. (2017). Performance calibration of low-cost and portable particular matter (PM) sensors. J. Aerosol Sci. 112: 1-10.

Manikonda, A., Zíková, N., Hopke, P.K. and Ferro, A.R. (2016). Laboratory assessment of low-cost PM monitors. J. Aerosol Sci. 102: 29-40.

Morawska, L., Thai, P.K., Liu, X., Asumadu-Sakyi, A., Ayoko, G., Bartonova, A., Bedini, A., Chai, F., Christensen, B. and Dunbabin, M. (2018). Applications of low-cost sensing technologies for air quality monitoring and exposure assessment: How far have they gone? Environ. Int. 116: 286-299.

Moreno-Rangel, A., Sharpe, T., Musau, F. and McGill, G. (2018). Field evaluation of a low-cost indoor air quality monitor to quantify exposure to pollutants in residential environments. J. Sens. Sens. Syst. 7: 373-388.

Papapostolou, V., Zhang, H., Feenstra, B.J. and Polidori, A. (2017). Development of an environmental chamber for evaluating the performance of low-cost air quality sensors under controlled conditions. Atmos. Environ. 171: 82-90.

Patel, S., Li, J., Pandey, A., Pervez, S., Chakrabarty, R.K. and Biswas, P. (2017). Spatio-temporal measurement of indoor particulate matter concentrations using a wireless network of low-cost sensors in households using solid fuels. Environ. Res. 152: 59-65.

Polidori, A., Papapostolou, V., Feenstra, B. and Zhang, H. (2017). Field evaluation of low-cost air quality sensors. South Coast Air Quality Management District, USA.

Pope III, C.A., Burnett, R.T., Thun, M.J., Calle, E.E., Krewski, D., Ito, K. and Thurston, G.D. (2002). Lung 
cancer, cardiopulmonary mortality, and long-term exposure to fine particulate air pollution. JAMA 287: 1132-1141.

Prabakar, J., Mohan, V. and Ravisankar, K. (2015). Evaluation of low cost particulate matter sensor for indoor air quality measurement. Int. J. Innovative Res. Sci. Eng. Technol. 4: 366-369.

Rai, A.C., Kumar, P., Pilla, F., Skouloudis, A.N., Di Sabatino, S., Ratti, C., Yasar, A. and Rickerby, D. (2017). End-user perspective of low-cost sensors for outdoor air pollution monitoring. Sci Total Environ. 607: 691-705.

Rajasegarar, S., Zhang, P., Zhou, Y., Karunasekera, S., Leckie, C. and Palaniswami, M. (2014). Long-term field evaluation of the plantower pms low-cost particulate matter sensors. 2014 IEEE Ninth International Conference on Intelligent Sensors, Sensor Networks and Information Processing (ISSNIP), 2014, Singapore, pp. 1-6.

Sayahi, T., Butterfield, A. and Kelly, K. (2019). Long-term field evaluation of the plantower pms low-cost particulate matter sensors. Environ. Pollut. 245: 932-940.

Semple, S., Ibrahim, A.E., Apsley, A., Steiner, M. and Turner, S. (2013). Using a new, low-cost air quality sensor to quantify second-hand smoke (SHS) levels in homes. Tob. Control 24: 153-158.

Singer, B.C. and Delp, W.W. (2018). Response of consumer and research grade indoor air quality monitors to residential sources of fine particles. Indoor Air 28: 624-639

Sioutas, C. (1999). Evaluation of the measurement performance of the scanning mobility particle sizer and aerodynamic particle sizer. Aerosol Sci.Technol. 30: 8492.

Smith, K.R. and Mehta, S. (2003). The burden of disease from indoor air pollution in developing countries: Comparison of estimates. Int. J. Hyg. Environ. Health 206: 279-289.

Sousan, S., Koehler, K., Hallett, L. and Peters, T.M. (2016a). Evaluation of the Alphasense optical particle counter (OPC-N2) and the Grimm portable aerosol spectrometer (PAS-1.108). Aerosol Sci. Technol. 50: 1352-1365.

Sousan, S., Koehler, K., Thomas, G., Park, J.H., Hillman, M., Halterman, A. and Peters, T.M. (2016b). Intercomparison of low-cost sensors for measuring the mass concentration of occupational aerosols. Aerosol Sci. Technol. 50: 462-473.
Sousan, S., Koehler, K., Hallett, L. and Peters, T.M. (2017). Evaluation of consumer monitors to measure particulate matter. J. Aerosol Sci. 107: 123-133.

Stewart, B. and Wild, C.P. (2017). World cancer report 2014. International Agency for Research on Cancer, WHO Press, World Health Organization, Switzerland.

U.S. EPA (2016). Technical assistance document for the reporting of daily air quality - the air quality index (AQI). U.S. Environmental Protection Agency, USA.

Valavanidis, A., Fiotakis, K. and Vlachogianni, T. (2008). Airborne particulate matter and human health: Toxicological assessment and importance of size and composition of particles for oxidative damage and carcinogenic mechanisms. J. Environ. Sci. Health, Part C 26: 339-362.

Wang, Y., Li, J., Jing, H., Zhang, Q., Jiang, J. and Biswas, P. (2015). Laboratory evaluation and calibration of three low-cost particle sensors for particulate matter measurement. Aerosol Sci. Technol. 49: 1063-1077.

Wang, Y., Pinterich, T. and Wang, J. (2018). Rapid measurement of sub-micrometer aerosol size distribution using a fast integrated mobility spectrometer. J. Aerosol Sci. 121: 12-20.

White, R.M., Paprotny, I., Doering, F., Cascio, W.E., Solomon, P.A. and Gundel, L.A. (2012). Sensors and apps for community-based atmospheric monitoring. EM: Air And Waste Management Association's Magazine for Environmental Managers. Air \& Waste Management Association, Pittsburgh, PA, pp 36-40.

World Health Organization (WHO) (2005). Who air quality guidelines for particulate matter, ozone, nitrogen dioxide and sulphur dioxide. Global update 2005. Summary of risk assessment. World Health Organization.

Zhang, J., Marto, J.P. and Schwab, J.J. (2018). Exploring the applicability and limitations of selected optical scattering instruments for PM mass measurement. Atmos. Meas. Tech. 11: 2995.

Zikova, N., Hopke, P.K. and Ferro, A.R. (2017). Evaluation of new low-cost particle monitors for $\mathrm{PM}_{2.5}$ concentrations measurements. J. Aerosol Sci. 105: 24-34.

Received for review, December 29, 2018 Revised, April 27, 2019 Accepted, July 1, 2019 